CRYSTALLOGRAPHIC COMMUNICATIONS

ISSN 2056-9890

Received 23 January 2016

Accepted 28 March 2016

Edited by W. T. A. Harrison, University of Aberdeen, Scotland

Keywords: crystal structure; 5-aminoisophthalic acid; 5AIA; 1,2-bis(pyridin-4-yl)ethene (BE); cocrystal; hydrogen bonding.

CCDC reference: 1471029

Supporting information: this article has supporting information at journals.iucr.org/e

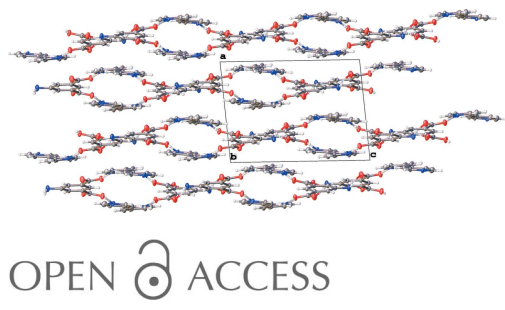

\section{Crystal structure of the co-crystal of 5-amino- isophthalic acid and 1,2-bis(pyridin-4-yl)ethene}

Scott C. McGuire, ${ }^{a}$ Steven C. Travis, ${ }^{a}$ Daniel W. Tuohey, ${ }^{a}$ Thomas J. Deering, ${ }^{\text {a }}$ Bob Martin, ${ }^{b}$ Jordan M. Cox ${ }^{\mathrm{a}}$ and Jason B. Benedict ${ }^{\mathrm{c} *}$

${ }^{\mathbf{a}} 764$ Natural Sciences Complex, Buffalo, 14260-3000, USA, b345 Natural Sciences Complex, Buffalo, 14260-3000,

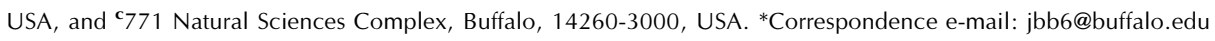

In the title $1: 1$ co-crystal, $\mathrm{C}_{12} \mathrm{H}_{10} \mathrm{~N}_{2} \cdot \mathrm{C}_{8} \mathrm{H}_{7} \mathrm{NO}_{4}$, the bipyridine moiety shows whole-molecule disorder over two sets of sites in a 0.588 (3): 0.412 (3) ratio. In the crystal, the components form hydrogen-bonded sheets linked by $\mathrm{N}-\mathrm{H} \cdots \mathrm{O}$ and $\mathrm{O}-\mathrm{H} \cdots \mathrm{N}$ interactions, which stack along the $a$ axis. A comparison to a related and previously published co-crystal of 5-amino-isophthalic acid and the shorter 4,4'-bipryidine is presented.

\section{Chemical context}

5-Amino-isophthalic acid (5AIA) is an emerging secondary building unit for a wide variety of metal-organic frameworks (MOFs). (Zeng et al., 2009; Wang et al., 2011; Cox et al., 2015) This compound is also a convenient precursor for the synthesis of azo-derivatized framework ligands, a key component in the rapidly evolving field of photochromic MOFs. (Brown et al., 2013; Castellanos et al., 2016; Walton et al., 2013; Patel et al., 2014). Similarly, 1,2-bis(pyridin-4-yl)ethene (BE) is also commonly used in MOF synthesis; however, it is routinely used in co-crystal engineering as well (Kongshaug \& Fjellvag, 2003; MacGillivray et al., 2008; Desiraju, 1995) The 5AIA-BE co-crystal presented herein was produced as part of an undergraduate physical chemistry laboratory experiment developed by Jason Benedict.<smiles>Nc1cc(C(=O)O)cc(C(=O)O)c1</smiles><smiles>C(=C/c1ccncc1)\c1ccncc1</smiles>

Recently, the co-crystal structure of 5AIA and 4,4'-bipyridine (BP), a shorter analogue of BE, was reported (Zhang et al., 2009). Unlike many MOFs in which different length linkers lead to isorecticular structures (Eddaoudi et al., 2002), the 5AIA-BP co-crystal exhibits several notable similarities and differences when compared to 5AIA-BE. As shown in Figs. 4, 5AIA forms hydrogen bonds with two 5AIA molecules and two BP molecules. The 5AIA-BP interactions and one of the 5AIA-5AIA interactions are similar to those found in 5AIA-BE. The remaining 5AIA-5AIA interaction in 5AIA$\mathrm{BP}$ consists solely of an $\mathrm{N}$ (amine) $-\mathrm{H} \cdots \mathrm{OH}$ hydrogen bond, as opposed to the $\mathrm{N}$ (amine) $-\mathrm{H} \cdots \mathrm{O}=\mathrm{C}$ interaction found in 5AIA-BP. Interestingly, this results in a total of five hydrogen 

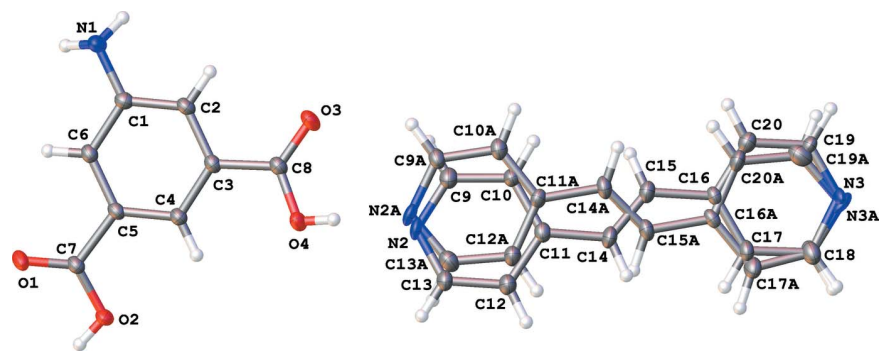

Figure 1

The asymmetric unit of the title compound, showing the numbering scheme. Displacement ellipsoids are shown at the $50 \%$ probability level.

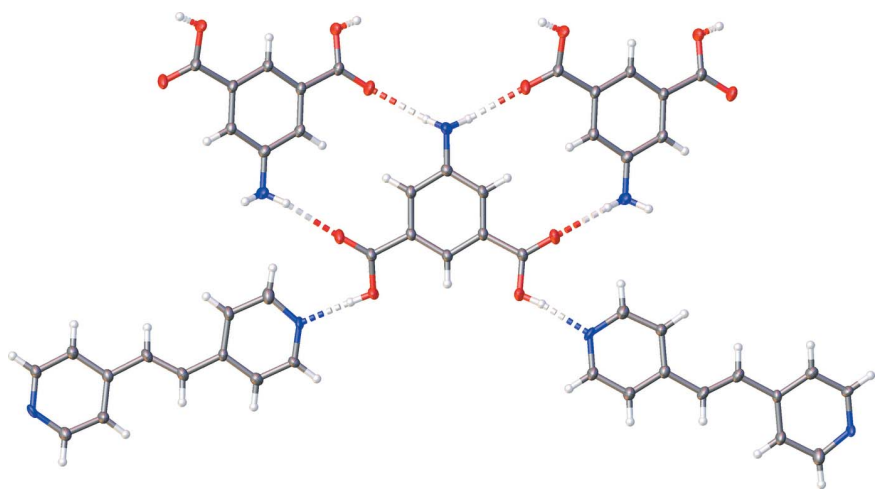

Figure 2

Diagram illustrating the hydrogen-bonding interactions present in the two-dimensional sheets found in the 5AIA-BE co-crystal.

bonds in the 5AIA-BP structure compared to the six hydrogen bonds observed in 5AIA-BE.

\section{Structural commentary}

The 5AIA-BE co-crystal crystallizes with one molecule of 5AIA and one molecule of BE in the asymmetric unit (Fig. 1). Both molecules are effectively planar in the solid state (r.m.s. deviation for 5AIA $=0.155 \AA$ ). The BE moiety shows whole
Table 1

Hydrogen-bond geometry $\left(\AA{ }^{\circ}\right)$.

\begin{tabular}{lllll}
\hline$D-\mathrm{H} \cdots A$ & $D-\mathrm{H}$ & $\mathrm{H} \cdots A$ & $D \cdots A$ & $D-\mathrm{H} \cdots A$ \\
\hline $\mathrm{N} 1-\mathrm{H} 1 A \cdots \mathrm{O} 1^{\mathrm{i}}$ & $0.899(17)$ & $2.062(17)$ & $2.9540(13)$ & $171.0(15)$ \\
$\mathrm{N} 1-\mathrm{H} 1 B \cdots 3^{\mathrm{ii}}$ & $0.894(17)$ & $2.157(17)$ & $3.0500(13)$ & $178.6(13)$ \\
$\mathrm{O} 2-\mathrm{H} 2 \cdots \mathrm{N} 3^{\text {ii }}$ & $0.989(19)$ & $1.70(2)$ & $2.688(8)$ & $173.4(18)$ \\
$\mathrm{O} 2-\mathrm{H} 2 \cdots \mathrm{N} 3 A^{\mathrm{iii}}$ & $0.989(19)$ & $1.63(2)$ & $2.619(12)$ & $177(2)$ \\
$\mathrm{O} 4-\mathrm{H} 4 \cdots \mathrm{N} 2^{\text {iv }}$ & $0.98(2)$ & $1.72(2)$ & $2.702(7)$ & $173.2(19)$ \\
$\mathrm{O} 4-\mathrm{H} 4 \cdots \mathrm{N} 2 A^{\text {iv }}$ & $0.98(2)$ & $1.59(2)$ & $2.566(11)$ & $175(2)$ \\
\hline
\end{tabular}

Symmetry codes: (i) $\quad-x+\frac{1}{2}, y-\frac{1}{2},-z+\frac{1}{2} ; \quad$ (ii) $\quad-x+\frac{1}{2}, y+\frac{1}{2},-z+\frac{1}{2}$;
$-x+1,-y+2,-z+1$; (iv) $x-\frac{1}{2},-y+\frac{1}{2}, z+\frac{1}{2}$.

molecule disorder over two sets of sites, consistent with a local $C 2$ rotation about the long axis of the molecule. The occupancy of the major and minor components was refined to be 0.588 (3) and 0.412 (3), respectively.

\section{Supramolecular features}

In this structure, the 5AIA molecule forms hydrogen bonds to both itself and the BE moiety, forming extended sheets (Table 1 and Fig. 2). The 5AIA-5AIA interactions consist of $\mathrm{N}$ (amine) $-\mathrm{H} \cdots \mathrm{O}=\mathrm{C}$ hydrogen bonds where each 5AIA makes two hydrogen bonds with two neighboring 5AIA molecules. The 5AIA-BE interaction consists of an $\mathrm{O}-$ $\mathrm{H} \cdots \mathrm{N}$ (pyridyl) hydrogen bond such that each 5AIA makes one hydrogen bond with two neighboring BE molecules. The sheets formed by these interactions stack along the the $a$ axis to produce a layered structure (Fig. 3).

\section{Database survey}

Recently, the co-crystal structure of 5AIA and 4,4'-bipyridine (BP), a shorter analogue of BE, was reported (Zhang et al., 2009). Unlike many MOFs in which different length linkers lead to isorecticular structures (Eddaoudi et al., 2002), the 5AIA-BP co-crystal exhibits several notable similarites and differences when compared to 5AIA-BE. As shown in Figs. 4,

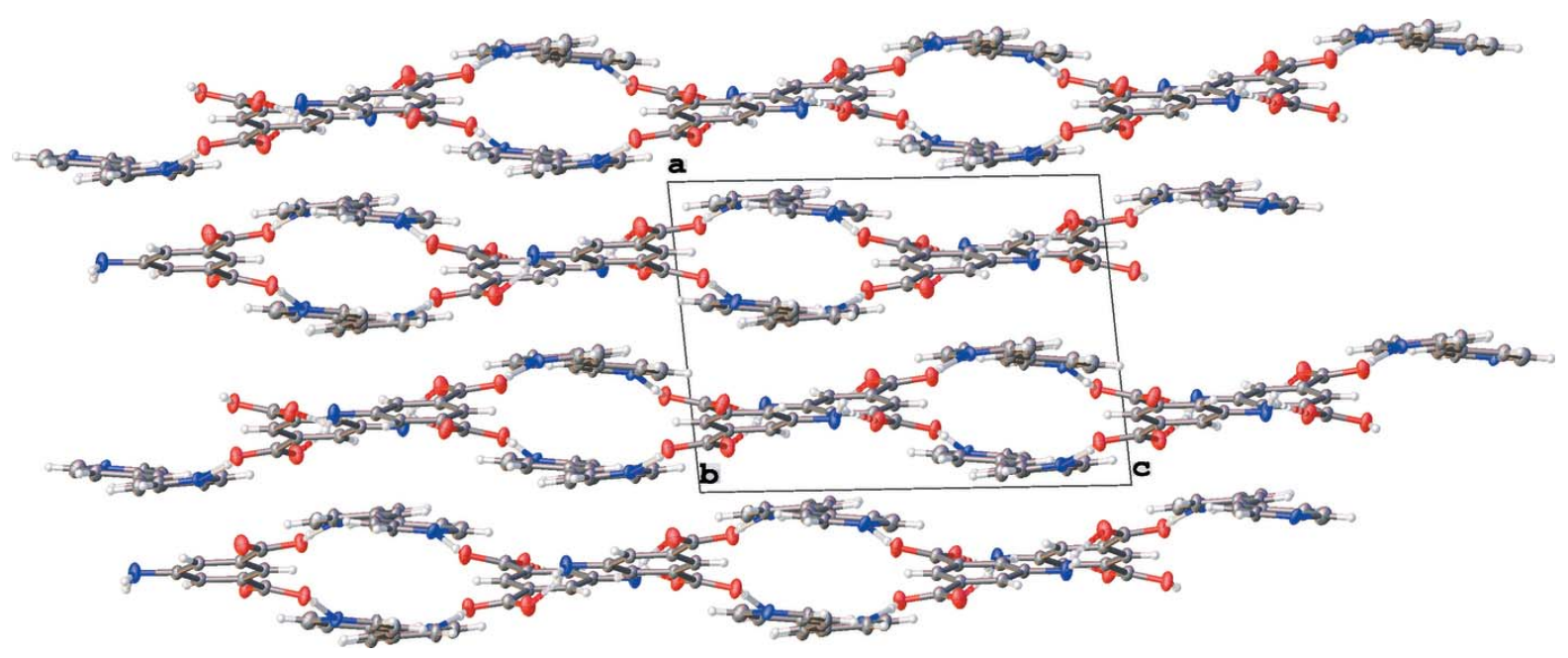

Figure 3

View down [001] showing the (100) sheets in the extended structure of the title compound. 


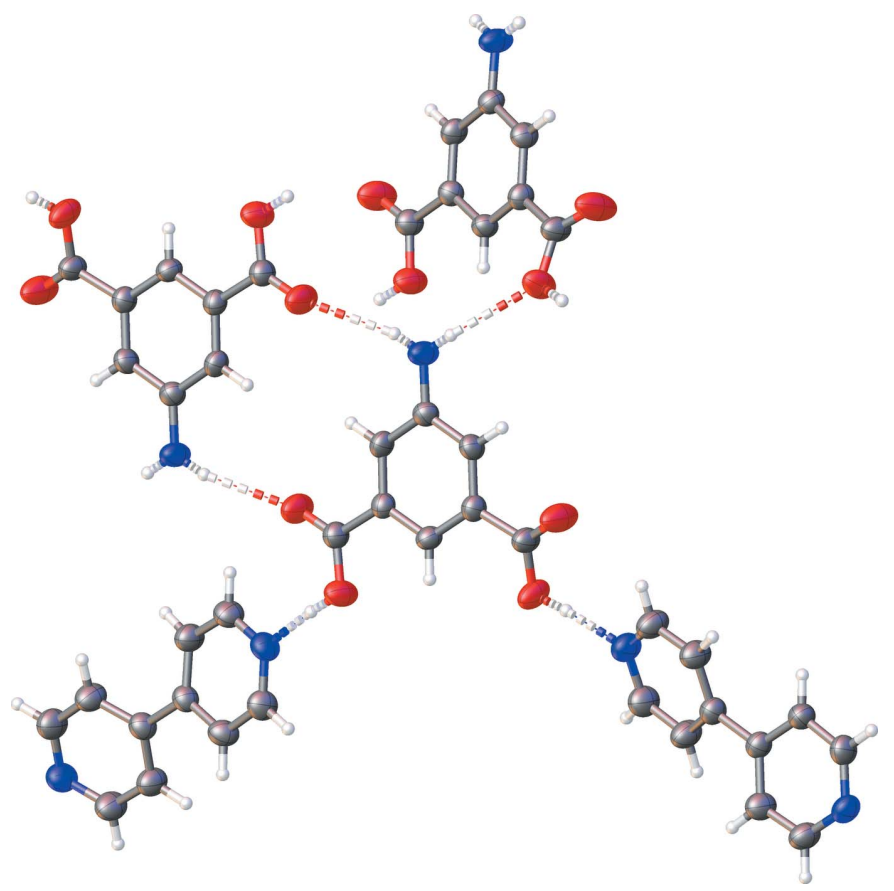

Figure 4

Diagram illustrating the hydrogen bonding interactions present in the previously reported 5AIA-BP co-crystal.

5AIA forms hydrogen bonds with two 5AIA molecules and two BP molecules. The 5AIA-BP interactions and one of the 5AIA-5AIA interactions are similar to those found in 5AIABE. The remaining 5AIA-5AIA interaction in 5AIA-BP consists solely of an $\mathrm{N}($ amine $)-\mathrm{H} \cdots \mathrm{OH}$ hydrogen bond, as opposed to the $\mathrm{N}($ amine $)-\mathrm{H} \cdots \mathrm{O}=\mathrm{C}$ interaction found in 5AIA-BP. Interestingly, this results in a total of five hydrogen bonds in the 5AIA-BP structure compared to the six hydrogen bonds observed in 5AIA-BE.

\section{Synthesis and crystallization}

Solid BE (0.0119 g, $\left.6.53 \times 10^{-5} \mathrm{~mol}\right)$ and 5AIA (0.0109 g, 6.02 $\left.\times 10^{-5} \mathrm{~mol}\right)$ were added to a $25 \mathrm{ml}$ scintillation vial. To this was added approximately $15 \mathrm{ml}$ of ethyl acetate followed by gentle heating. An additional $2 \mathrm{ml}$ of methanol was added and all remaining solids dissolved. The loosely capped vial was then placed into a dark cabinet. After two weeks, yellow block-shaped crystals of the title compound suitable for singlecrystal X-ray diffraction measurements were obtained.

\section{Refinement}

Crystal data, data collection and structure refinement details are summarized in Table 2. Heteroatom hydrogen atoms were located in difference electron-density maps and freely refined. Hydrogen atoms attached to carbon atoms were refined using riding models with $\mathrm{C}-\mathrm{H}=0.95 \AA$ and $U_{\text {iso }}(\mathrm{H})=1.2 U_{\text {eq }}(\mathrm{C})$. The BE was found to be disordered over two sets of sites in a 0.588 (3): 0.412 (3) ratio.
Table 2

Experimental details.

\begin{tabular}{|c|c|}
\hline \multicolumn{2}{|l|}{ Crystal data } \\
\hline Chemical formula & $\mathrm{C}_{12} \mathrm{H}_{10} \mathrm{~N}_{2} \cdot \mathrm{C}_{8} \mathrm{H}_{7} \mathrm{NO}_{4}$ \\
\hline$M_{\mathrm{r}}$ & 363.36 \\
\hline Crystal system, space group & Monoclinic, $P 2_{1} / n$ \\
\hline Temperature (K) & 90 \\
\hline$a, b, c(\AA)$ & $\begin{array}{l}10.1614(10), 12.0782(12), \\
\quad 14.0537(14)\end{array}$ \\
\hline$\beta\left({ }^{\circ}\right)$ & $95.027(2)$ \\
\hline$V\left(\AA^{6}\right)$ & $1718.2(3)$ \\
\hline$Z$ & 4 \\
\hline Radiation type & Мo $K \alpha$ \\
\hline$\mu\left(\mathrm{mm}^{-1}\right)$ & 0.10 \\
\hline Crystal size $(\mathrm{mm})$ & $0.22 \times 0.2 \times 0.18$ \\
\hline \multicolumn{2}{|l|}{ Data collection } \\
\hline Diffractometer & Bruker SMART APEXII CCD \\
\hline Absorption correction & $\begin{array}{l}\text { Multi-scan (SADABS; Bruker, } \\
\text { 2014) }\end{array}$ \\
\hline$T_{\min }, T_{\max }$ & $0.683,0.747$ \\
\hline $\begin{array}{l}\text { No. of measured, independent and } \\
\text { observed }[I>2 \sigma(I)] \text { reflections }\end{array}$ & $24372,6546,4519$ \\
\hline$R_{\text {int }}$ & 0.033 \\
\hline$(\sin \theta / \lambda)_{\max }\left(\AA^{-1}\right)$ & 0.771 \\
\hline \multicolumn{2}{|l|}{ Refinement } \\
\hline$R\left[F^{2}>2 \sigma\left(F^{2}\right)\right], w R\left(F^{2}\right), S$ & $0.047,0.143,1.02$ \\
\hline No. of reflections & 6546 \\
\hline No. of parameters & 378 \\
\hline $\mathrm{H}$-atom treatment & $\begin{array}{l}\mathrm{H} \text { atoms treated by a mixture of } \\
\text { independent and constrained } \\
\text { refinement }\end{array}$ \\
\hline$\Delta \rho_{\max }, \Delta \rho_{\min }\left(\mathrm{e} \AA^{-3}\right)$ & $0.40,-0.24$ \\
\hline
\end{tabular}

Computer programs: APEX2 and SAINT (Bruker, 2014), SHELXT (Sheldrick, 2015), SHELXL2014 (Sheldrick, 2015b) and OLEX2 (Dolomanov et al., 2009).

\section{Acknowledgements}

This material is based upon work supported by the National Science Foundation under grant No. DMR-1455039.

\section{References}

Brown, J. W., Henderson, B. L., Kiesz, M. D., Whalley, A. C., Morris, W., Grunder, S., Deng, H., Furukawa, H., Zink, J. I., Stoddart, J. F. \& Yaghi, O. M. (2013). Chem. Sci. 4, 2858-2864.

Bruker (2014). APEX2, SAINT and SADABS. Bruker AXS Inc., Madison, Wisconsin, USA.

Castellanos, S., Goulet-Hanssens, A., Zhao, F., Dikhtiarenko, A., Pustovarenko, A., Hecht, S., Gascon, J., Kapteijn, F. \& Bléger, D. (2016). Chem. Eur. J. 22, 746-752.

Cox, J. M., Walton, I. M., Benson, C. A., Chen, Y.-S. \& Benedict, J. B. (2015). J. Appl. Cryst. 48, 578-581.

Desiraju, G. R. (1995). Angew. Chem. Int. Ed. Engl. 34, 23112327.

Dolomanov, O. V., Bourhis, L. J., Gildea, R. J., Howard, J. A. K. \& Puschmann, H. (2009). J. Appl. Cryst. 42, 339-341.

Eddaoudi, M., Kim, J., Rosi, N., Vodak, D., Wachter, J., O'Keeffe, M. \& Yaghi, O. M. (2002). Science, 295, 469-472.

Kongshaug, K. O. \& Fjellvåg, H. (2003). J. Solid State Chem. 175, 182187.

MacGillivray, L. R., Papaefstathiou, G. S., Friščić, T., Hamilton, T. D., Bučar, D.-K., Chu, Q., Varshney, D. B. \& Georgiev, I. G. (2008). Acc. Chem. Res. 41, 280-291.

Patel, D. G. (D.), Walton, I. M., Cox, J. M., Gleason, C. J., Butzer, D. R. \& Benedict, J. B. (2014). Chem. Commun. 50, 2653-2656.

Sheldrick, G. M. (2015). Acta Cryst. C71, 3-8. 


\section{research communications}

Walton, I. M., Cox, J. M., Coppin, J. A., Linderman, C. M., Patel, D. G. (D.) \& Benedict, J. B. (2013). Chem. Commun. 49, 8012-8014.

Wang, H.-N., Meng, X., Yang, G.-S., Wang, X.-L., Shao, K.-Z., Su, Z.-M. \& Wang, C.-G. (2011). Chem. Commun. 47, 7128-7130.
Zeng, M.-H., Hu, S., Chen, Q., Xie, G., Shuai, Q., Gao, S.-L. \& Tang, L.-Y. (2009). Inorg. Chem. 48, 7070-7079.

Zhang, X., Zhu, B. \& Guo, F. (2009). Asian J. Chem. 21, 7072-7076. 


\section{supporting information}

Acta Cryst. (2016). E72, 639-642［doi:10.1107/S2056989016005259]

\section{Crystal structure of the co-crystal of 5-aminoisophthalic acid and 1,2-bis- (pyridin-4-yl)ethene}

\section{Scott C. McGuire, Steven C. Travis, Daniel W. Tuohey, Thomas J. Deering, Bob Martin, Jordan}

\section{Cox and Jason B. Benedict}

\section{Computing details}

Data collection: APEX2 (Bruker, 2014); cell refinement: SAINT (Bruker, 2014); data reduction: SAINT (Bruker, 2014); program(s) used to solve structure: SHELXT (Sheldrick, 2015); program(s) used to refine structure: SHELXL2014 (Sheldrick, 2015b); molecular graphics: OLEX2 (Dolomanov et al., 2009).

5-Aminoisophthalic acid-1,2-bis(pyridin-4-yl)ethene (1/1)

Crystal data

$\mathrm{C}_{12} \mathrm{H}_{10} \mathrm{~N}_{2} \cdot \mathrm{C}_{8} \mathrm{H}_{7} \mathrm{NO}_{4}$

$M_{r}=363.36$

Monoclinic, $P 2_{1} / n$

$a=10.1614(10) \AA$

$b=12.0782(12) \AA$

$c=14.0537$ (14) $\AA$

$\beta=95.027(2)^{\circ}$

$V=1718.2(3) \AA^{3}$

$Z=4$

Data collection

Bruker SMART APEXII CCD diffractometer

Radiation source: microfocus rotating anode, Incoatec I $\mu \mathrm{s}$

Mirror optics monochromator

Detector resolution: 7.9 pixels $\mathrm{mm}^{-1}$

$\omega$ scans

Absorption correction: multi-scan

(SADABS; Bruker, 2014)

\section{Refinement}

Refinement on $F^{2}$

Least-squares matrix: full

$R\left[F^{2}>2 \sigma\left(F^{2}\right)\right]=0.047$

$w R\left(F^{2}\right)=0.143$

$S=1.02$

6546 reflections

378 parameters

0 restraints
$F(000)=760$

$D_{\mathrm{x}}=1.405 \mathrm{Mg} \mathrm{m}^{-3}$

Mo $K \alpha$ radiation, $\lambda=0.71073 \AA$

Cell parameters from 428 reflections

$\theta=2.8-22.0^{\circ}$

$\mu=0.10 \mathrm{~mm}^{-1}$

$T=90 \mathrm{~K}$

Block, yellow

$0.22 \times 0.2 \times 0.18 \mathrm{~mm}$

$T_{\min }=0.683, T_{\max }=0.747$

24372 measured reflections

6546 independent reflections

4519 reflections with $I>2 \sigma(I)$

$R_{\text {int }}=0.033$

$\theta_{\text {max }}=33.2^{\circ}, \theta_{\min }=2.2^{\circ}$

$h=-15 \rightarrow 15$

$k=-16 \rightarrow 18$

$l=-19 \rightarrow 21$

Hydrogen site location: mixed

$\mathrm{H}$ atoms treated by a mixture of independent and constrained refinement

$w=1 /\left[\sigma^{2}\left(F_{\mathrm{o}}^{2}\right)+(0.0727 P)^{2}+0.2884 P\right]$

where $P=\left(F_{\mathrm{o}}^{2}+2 F_{\mathrm{c}}^{2}\right) / 3$

$(\Delta / \sigma)_{\max }<0.001$

$\Delta \rho_{\max }=0.40 \mathrm{e} \AA^{-3}$

$\Delta \rho_{\min }=-0.24$ e $\AA^{-3}$ 


\section{Special details}

Geometry. All esds (except the esd in the dihedral angle between two 1.s. planes) are estimated using the full covariance matrix. The cell esds are taken into account individually in the estimation of esds in distances, angles and torsion angles; correlations between esds in cell parameters are only used when they are defined by crystal symmetry. An approximate (isotropic) treatment of cell esds is used for estimating esds involving l.s. planes.

Fractional atomic coordinates and isotropic or equivalent isotropic displacement parameters $\left(\AA^{2}\right)$

\begin{tabular}{|c|c|c|c|c|c|}
\hline & $x$ & $y$ & $z$ & $U_{\text {iso }} * / U_{\text {eq }}$ & Occ. $(<1)$ \\
\hline O1 & $0.21821(10)$ & $0.91797(6)$ & $0.42759(6)$ & $0.0299(2)$ & \\
\hline $\mathrm{O} 2$ & $0.18838(9)$ & $0.81890(6)$ & $0.55840(5)$ & $0.02654(18)$ & \\
\hline $\mathrm{H} 2$ & $0.1646(19)$ & $0.8932(16)$ & $0.5810(13)$ & $0.058(5)^{*}$ & \\
\hline $\mathrm{O} 3$ & $0.35542(10)$ & $0.33662(6)$ & $0.42624(6)$ & $0.0309(2)$ & \\
\hline $\mathrm{O} 4$ & $0.35891(9)$ & $0.43117(6)$ & $0.56369(5)$ & $0.02640(18)$ & \\
\hline H4 & $0.388(2)$ & $0.3606(16)$ & $0.5933(14)$ & $0.064(6)^{*}$ & \\
\hline N1 & $0.27113(12)$ & $0.63283(8)$ & $0.17186(7)$ & $0.0291(2)$ & \\
\hline H1A & $0.2656(17)$ & $0.5692(14)$ & $0.1385(12)$ & $0.043(4)^{*}$ & \\
\hline H1B & $0.2351(16)$ & $0.6925(14)$ & $0.1424(11)$ & $0.040(4)^{*}$ & \\
\hline $\mathrm{C} 1$ & $0.27076(11)$ & $0.62966(8)$ & $0.26981(7)$ & $0.02010(19)$ & \\
\hline $\mathrm{C} 2$ & $0.29909(11)$ & $0.53166(8)$ & $0.32097(7)$ & $0.02032(19)$ & \\
\hline $\mathrm{H} 2 \mathrm{~A}$ & 0.3164 & 0.4657 & 0.2874 & $0.024^{*}$ & \\
\hline $\mathrm{C} 3$ & $0.30223(10)$ & $0.52951(8)$ & $0.42012(7)$ & $0.01921(19)$ & \\
\hline $\mathrm{C} 4$ & $0.27368(11)$ & $0.62462(8)$ & $0.47130(7)$ & $0.01990(19)$ & \\
\hline $\mathrm{H} 4 \mathrm{~A}$ & 0.2728 & 0.6226 & 0.5388 & $0.024 *$ & \\
\hline $\mathrm{C} 5$ & $0.24644(10)$ & $0.72289(8)$ & $0.42033(7)$ & $0.01881(19)$ & \\
\hline C6 & $0.24657(10)$ & $0.72561(8)$ & $0.32144(7)$ & 0.01947 (19) & \\
\hline H6 & 0.2300 & 0.7935 & 0.2884 & $0.023^{*}$ & \\
\hline $\mathrm{C} 7$ & $0.21669(11)$ & $0.82903(8)$ & $0.46877(7)$ & $0.0211(2)$ & \\
\hline $\mathrm{C} 8$ & $0.34070(11)$ & $0.42315(8)$ & $0.46985(7)$ & $0.0215(2)$ & \\
\hline $\mathrm{C} 18$ & $0.89056(13)$ & $0.89912(9)$ & $0.43551(9)$ & $0.0306(3)$ & \\
\hline H18 & 0.8831 & 0.9091 & 0.5019 & $0.037^{*}$ & $0.588(3)$ \\
\hline $\mathrm{H} 18 \mathrm{~A}$ & 0.8865 & 0.9167 & 0.5011 & $0.037^{*}$ & $0.412(3)$ \\
\hline N2 & $0.9309(8)$ & $0.2578(6)$ & $0.1571(3)$ & $0.0206(8)$ & $0.588(3)$ \\
\hline N3 & $0.8888(10)$ & $0.9864(5)$ & $0.3724(9)$ & $0.0203(11)$ & $0.588(3)$ \\
\hline C9 & $0.9283(3)$ & $0.3491(2)$ & $0.1046(2)$ & $0.0259(5)$ & $0.588(3)$ \\
\hline H9 & 0.9195 & 0.3426 & 0.0370 & $0.031^{*}$ & $0.588(3)$ \\
\hline $\mathrm{C} 10$ & $0.9382(2)$ & $0.45426(16)$ & $0.1453(2)$ & $0.0246(4)$ & $0.588(3)$ \\
\hline H10 & 0.9393 & 0.5181 & 0.1058 & $0.029^{*}$ & $0.588(3)$ \\
\hline $\mathrm{C} 11$ & $0.94634(18)$ & $0.46501(16)$ & $0.24375(18)$ & $0.0202(4)$ & $0.588(3)$ \\
\hline $\mathrm{C} 12$ & $0.9527(2)$ & $0.36771(18)$ & $0.29764(18)$ & $0.0260(5)$ & $0.588(3)$ \\
\hline H12 & 0.9624 & 0.3709 & 0.3654 & $0.031^{*}$ & $0.588(3)$ \\
\hline $\mathrm{C} 13$ & $0.9446(4)$ & $0.2666(3)$ & $0.2511(2)$ & $0.0240(6)$ & $0.588(3)$ \\
\hline H13 & 0.9491 & 0.2008 & 0.2883 & $0.029^{*}$ & $0.588(3)$ \\
\hline $\mathrm{C} 14$ & $0.9425(2)$ & $0.57258(15)$ & $0.29274(13)$ & $0.0245(5)$ & $0.588(3)$ \\
\hline H14 & 0.9538 & 0.5724 & 0.3606 & $0.029^{*}$ & $0.588(3)$ \\
\hline $\mathrm{C} 15$ & $0.9246(2)$ & $0.66976(15)$ & $0.24941(15)$ & $0.0241(4)$ & $0.588(3)$ \\
\hline H15 & 0.9170 & 0.6692 & 0.1816 & $0.029 *$ & $0.588(3)$ \\
\hline C16 & $0.9152(3)$ & $0.7790(2)$ & $0.2963(2)$ & $0.0182(5)$ & $0.588(3)$ \\
\hline
\end{tabular}




$\begin{array}{llllll}\text { C17 } & 0.9045(7) & 0.7916(5) & 0.3934(2) & 0.0242(8) & 0.588(3) \\ \text { H17 } & 0.9064 & 0.7278 & 0.4330 & 0.029^{*} & 0.588(3) \\ \text { C19 } & 0.9010(9) & 0.9740(6) & 0.2814(5) & 0.0214(8) & 0.588(3) \\ \text { H19 } & 0.8997 & 1.0381 & 0.2422 & 0.026^{*} & 0.588(3) \\ \text { C20 } & 0.9153(4) & 0.8733(3) & 0.2402(3) & 0.0244(6) & 0.588(3) \\ \text { H20 } & 0.9254 & 0.8678 & 0.1738 & 0.029^{*} & 0.588(3) \\ \text { C17A } & 0.9027(10) & 0.7907(7) & 0.4220(4) & 0.0258(10) & 0.412(3) \\ \text { H17A } & 0.9026 & 0.7388 & 0.4728 & 0.031^{*} & 0.412(3) \\ \text { C9A } & 0.9486(4) & 0.3433(3) & 0.0717(3) & 0.0219(7) & 0.412(3) \\ \text { H9A } & 0.9550 & 0.3259 & 0.0064 & 0.026^{*} & 0.412(3) \\ \text { C20A } & 0.9161(6) & 0.8429(4) & 0.2607(4) & 0.0260(11) & 0.412(3) \\ \text { H20A } & 0.9275 & 0.8265 & 0.1958 & 0.031^{*} & 0.412(3) \\ \text { N2A } & 0.9319(11) & 0.2579(10) & 0.1328(4) & 0.0202(10) & 0.412(3) \\ \text { N3A } & 0.8830(15) & 0.9861(9) & 0.3829(13) & 0.025(2) & 0.412(3) \\ \text { C19A } & 0.8995(15) & 0.9532(9) & 0.2902(9) & 0.034(2) & 0.412(3) \\ \text { H19A } & 0.8996 & 1.0094 & 0.2428 & 0.040^{*} & 0.412(3) \\ \text { C10A } & 0.9569(3) & 0.4532(2) & 0.0982(3) & 0.0243(6) & 0.412(3) \\ \text { H10A } & 0.9663 & 0.5087 & 0.0514 & 0.029^{*} & 0.412(3) \\ \text { C11A } & 0.9515(3) & 0.4826(2) & 0.1934(3) & 0.0195(6) & 0.412(3) \\ \text { C12A } & 0.9434(3) & 0.3974(3) & 0.2586(3) & 0.0250(6) & 0.412(3) \\ \text { H12A } & 0.9448 & 0.4125 & 0.3250 & 0.030^{*} & 0.412(3) \\ \text { C13A } & 0.9330(6) & 0.2880(4) & 0.2245(4) & 0.0293(10) & 0.412(3) \\ \text { H13A } & 0.9262 & 0.2309 & 0.2703 & 0.035^{*} & 0.412(3) \\ \text { C14A } & 0.9493(3) & 0.6007(2) & 0.21878(19) & 0.0235(6) & 0.412(3) \\ \text { H14A } & 0.9676 & 0.6526 & 0.1709 & 0.028^{*} & 0.412(3) \\ \text { C15A } & 0.9237(3) & 0.6405(2) & 0.3033(2) & 0.0226(6) & 0.412(3) \\ \text { H15A } & 0.9095 & 0.5884 & 0.3521 & 0.027^{*} & 0.412(3) \\ \text { C16A } & 0.9155(4) & 0.7585(4) & 0.3276(3) & 0.0186(7) & 0.412(3)\end{array}$

Atomic displacement parameters $\left(\AA^{2}\right)$

\begin{tabular}{lllllll}
\hline & $U^{11}$ & $U^{22}$ & $U^{33}$ & $U^{12}$ & $U^{13}$ & $U^{23}$ \\
\hline O1 & $0.0508(6)$ & $0.0126(3)$ & $0.0286(4)$ & $0.0008(3)$ & $0.0170(4)$ & $-0.0002(3)$ \\
O2 & $0.0459(5)$ & $0.0155(3)$ & $0.0195(3)$ & $0.0059(3)$ & $0.0096(3)$ & $-0.0016(3)$ \\
O3 & $0.0510(6)$ & $0.0143(3)$ & $0.0270(4)$ & $0.0041(3)$ & $0.0012(4)$ & $-0.0046(3)$ \\
O4 & $0.0438(5)$ & $0.0149(3)$ & $0.0201(3)$ & $0.0063(3)$ & $0.0006(3)$ & $-0.0003(3)$ \\
N1 & $0.0510(7)$ & $0.0180(4)$ & $0.0186(4)$ & $0.0067(4)$ & $0.0058(4)$ & $-0.0018(3)$ \\
C1 & $0.0246(5)$ & $0.0175(4)$ & $0.0187(4)$ & $0.0010(4)$ & $0.0046(4)$ & $-0.0022(3)$ \\
C2 & $0.0255(5)$ & $0.0148(4)$ & $0.0210(4)$ & $0.0017(4)$ & $0.0039(4)$ & $-0.0038(3)$ \\
C3 & $0.0242(5)$ & $0.0130(4)$ & $0.0206(4)$ & $0.0003(3)$ & $0.0030(4)$ & $-0.0012(3)$ \\
C4 & $0.0269(5)$ & $0.0142(4)$ & $0.0191(4)$ & $0.0002(4)$ & $0.0047(4)$ & $-0.0016(3)$ \\
C5 & $0.0235(5)$ & $0.0130(4)$ & $0.0206(4)$ & $-0.0001(3)$ & $0.0056(4)$ & $-0.0026(3)$ \\
C6 & $0.0236(5)$ & $0.0140(4)$ & $0.0213(4)$ & $0.0010(3)$ & $0.0050(4)$ & $-0.0006(3)$ \\
C7 & $0.0285(5)$ & $0.0142(4)$ & $0.0214(4)$ & $0.0004(4)$ & $0.0069(4)$ & $-0.0025(3)$ \\
C8 & $0.0285(5)$ & $0.0145(4)$ & $0.0217(5)$ & $-0.0002(4)$ & $0.0025(4)$ & $-0.0023(3)$ \\
C18 & $0.0394(7)$ & $0.0178(5)$ & $0.0360(6)$ & $0.0017(4)$ & $0.0118(5)$ & $-0.0001(4)$ \\
N2 & $0.0261(11)$ & $0.0147(9)$ & $0.021(2)$ & $-0.0007(7)$ & $0.0014(18)$ & $-0.0088(18)$ \\
N3 & $0.0306(19)$ & $0.0099(16)$ & $0.022(3)$ & $0.0050(11)$ & $0.0112(14)$ & $-0.0001(12)$
\end{tabular}




$\begin{array}{lllllll}\text { C9 } & 0.0351(14) & 0.0194(9) & 0.0238(13) & -0.0024(8) & 0.0066(10) & -0.0027(10) \\ \text { C10 } & 0.0400(12) & 0.0158(8) & 0.0182(11) & -0.0012(7) & 0.0044(9) & -0.0006(8) \\ \text { C11 } & 0.0232(9) & 0.0158(10) & 0.0219(11) & -0.0012(6) & 0.0037(7) & -0.0024(7) \\ \text { C12 } & 0.0388(12) & 0.0170(9) & 0.0225(10) & 0.0016(8) & 0.0038(9) & -0.0012(8) \\ \text { C13 } & 0.0341(13) & 0.0160(13) & 0.0225(15) & 0.0007(10) & 0.0055(12) & -0.0034(9) \\ \text { C14 } & 0.0348(11) & 0.0173(9) & 0.0218(8) & 0.0012(7) & 0.0053(7) & -0.0064(6) \\ \text { C15 } & 0.0325(10) & 0.0176(8) & 0.0223(9) & -0.0004(7) & 0.0021(7) & -0.0056(7) \\ \text { C16 } & 0.0226(9) & 0.0109(14) & 0.0211(13) & 0.0001(8) & 0.0021(10) & 0.0004(10) \\ \text { C17 } & 0.0346(13) & 0.0133(9) & 0.026(2) & 0.0011(8) & 0.0086(18) & -0.0038(17) \\ \text { C19 } & 0.0301(15) & 0.0192(19) & 0.0155(15) & 0.0019(14) & 0.0054(11) & -0.0047(15) \\ \text { C20 } & 0.0324(12) & 0.0204(16) & 0.0205(14) & 0.0012(12) & 0.0019(10) & -0.0034(10) \\ \text { C17A } & 0.0330(18) & 0.0195(15) & 0.027(3) & 0.0003(12) & 0.011(3) & -0.005(2) \\ \text { C9A } & 0.0281(17) & 0.0136(11) & 0.0247(18) & -0.0015(10) & 0.0065(14) & -0.0010(13) \\ \text { C20A } & 0.0378(18) & 0.019(3) & 0.021(2) & 0.0028(19) & 0.0022(17) & -0.0085(17) \\ \text { N2A } & 0.0234(15) & 0.0201(13) & 0.017(3) & 0.0010(10) & -0.001(2) & -0.010(2) \\ \text { N3A } & 0.026(3) & 0.033(4) & 0.016(3) & -0.001(2) & 0.008(2) & -0.011(2) \\ \text { C19A } & 0.039(3) & 0.029(4) & 0.032(3) & -0.002(3) & 0.0015(19) & 0.006(2) \\ \text { C10A } & 0.0320(15) & 0.0166(11) & 0.0243(15) & -0.0019(10) & 0.0035(12) & -0.0024(10) \\ \text { C11A } & 0.0250(13) & 0.0163(11) & 0.0170(15) & -0.0012(9) & 0.0008(10) & -0.0038(11) \\ \text { C12A } & 0.0386(17) & 0.0155(16) & 0.0209(15) & 0.0010(11) & 0.0017(12) & -0.0020(11) \\ \text { C13A } & 0.041(2) & 0.019(2) & 0.027(3) & 0.0004(15) & 0.0012(19) & 0.0024(15) \\ \text { C14A } & 0.0299(14) & 0.0125(10) & 0.0283(13) & -0.0003(9) & 0.0033(10) & -0.0035(9) \\ \text { C15A } & 0.0308(14) & 0.0119(12) & 0.0251(14) & 0.0007(9) & 0.0020(10) & -0.0013(9) \\ \text { C16A } & 0.0207(13) & 0.0107(14) & 0.024(2) & 0.0002(10) & 0.0012(14) & 0.0033(15) \\ & & & & & & \end{array}$

Geometric parameters $\left(\AA,{ }^{\circ}\right)$

\begin{tabular}{llll}
\hline $\mathrm{O} 1-\mathrm{C} 7$ & $1.2209(12)$ & $\mathrm{C} 12-\mathrm{H} 12$ & 0.9500 \\
$\mathrm{O} 2-\mathrm{H} 2$ & $0.990(19)$ & $\mathrm{C} 12-\mathrm{C} 13$ & $1.385(4)$ \\
$\mathrm{O} 2-\mathrm{C} 7$ & $1.3222(12)$ & $\mathrm{C} 13-\mathrm{H} 13$ & 0.9500 \\
$\mathrm{O} 3-\mathrm{C} 8$ & $1.2274(12)$ & $\mathrm{C} 14-\mathrm{H} 14$ & 0.9500 \\
$\mathrm{O} 4-\mathrm{H} 4$ & $0.98(2)$ & $\mathrm{C} 14-\mathrm{C} 15$ & $1.327(3)$ \\
$\mathrm{O} 4-\mathrm{C} 8$ & $1.3191(12)$ & $\mathrm{C} 15-\mathrm{H} 15$ & 0.9500 \\
$\mathrm{~N} 1-\mathrm{H} 1 \mathrm{~A}$ & $0.899(17)$ & $\mathrm{C} 15-\mathrm{C} 16$ & $1.482(3)$ \\
$\mathrm{N} 1-\mathrm{H} 1 \mathrm{~B}$ & $0.894(17)$ & $\mathrm{C} 16-\mathrm{C} 17$ & $1.387(4)$ \\
$\mathrm{N} 1-\mathrm{C} 1$ & $1.3775(13)$ & $\mathrm{C} 16-\mathrm{C} 20$ & $1.385(3)$ \\
$\mathrm{C} 1-\mathrm{C} 2$ & $1.4017(14)$ & $\mathrm{C} 17-\mathrm{H} 17$ & 0.9500 \\
$\mathrm{C} 1-\mathrm{C} 6$ & $1.4005(13)$ & $\mathrm{C} 19-\mathrm{H} 19$ & 0.9500 \\
$\mathrm{C} 2-\mathrm{H} 2 \mathrm{~A}$ & 0.9500 & $\mathrm{C} 19-\mathrm{C} 20$ & $1.360(7)$ \\
$\mathrm{C} 2-\mathrm{C} 3$ & $1.3912(14)$ & $\mathrm{C} 20-\mathrm{H} 20$ & 0.9500 \\
$\mathrm{C} 3-\mathrm{C} 4$ & $1.3991(13)$ & $\mathrm{C} 17 \mathrm{~A}-\mathrm{H} 17 \mathrm{~A}$ & 0.9500 \\
$\mathrm{C} 3-\mathrm{C} 8$ & $1.4979(14)$ & $\mathrm{C} 17 \mathrm{~A}-\mathrm{C} 16 \mathrm{~A}$ & $1.399(6)$ \\
$\mathrm{C} 4-\mathrm{H} 4 \mathrm{~A}$ & 0.9500 & $\mathrm{C} 9 \mathrm{~A}-\mathrm{H} 9 \mathrm{~A}$ & 0.9500 \\
$\mathrm{C} 4-\mathrm{C} 5$ & $1.4014(13)$ & $\mathrm{C} 9 \mathrm{~A}-\mathrm{N} 2 \mathrm{~A}$ & $1.361(10)$ \\
$\mathrm{C} 5-\mathrm{C} 6$ & $1.3902(14)$ & $\mathrm{C} 9 \mathrm{~A}-\mathrm{C} 10 \mathrm{~A}$ & $1.379(4)$ \\
$\mathrm{C} 5-\mathrm{C} 7$ & $1.4948(13)$ & $\mathrm{C} 20 \mathrm{~A}-\mathrm{H} 20 \mathrm{~A}$ & 0.9500 \\
$\mathrm{C} 6-\mathrm{H} 6$ & 0.9500 & $\mathrm{C} 20 \mathrm{~A}-\mathrm{C} 19 \mathrm{~A}$ & $1.410(11)$ \\
$\mathrm{C} 18-\mathrm{H} 18$ & 0.9500 & $\mathrm{C} 20 \mathrm{~A}-\mathrm{C} 16 \mathrm{~A}$ & $1.388(5)$
\end{tabular}




\begin{tabular}{|c|c|c|c|}
\hline $\mathrm{C} 18-\mathrm{H} 18 \mathrm{~A}$ & 0.9500 & $\mathrm{~N} 2 \mathrm{~A}-\mathrm{C} 13 \mathrm{~A}$ & $1.338(7)$ \\
\hline $\mathrm{C} 18-\mathrm{N} 3$ & $1.376(9)$ & $\mathrm{N} 3 \mathrm{~A}-\mathrm{C} 19 \mathrm{~A}$ & $1.39(2)$ \\
\hline $\mathrm{C} 18-\mathrm{C} 17$ & $1.439(5)$ & $\mathrm{C} 19 \mathrm{~A}-\mathrm{H} 19 \mathrm{~A}$ & 0.9500 \\
\hline $\mathrm{C} 18-\mathrm{C} 17 \mathrm{~A}$ & $1.331(9)$ & $\mathrm{C} 10 \mathrm{~A}-\mathrm{H} 10 \mathrm{~A}$ & 0.9500 \\
\hline $\mathrm{C} 18-\mathrm{N} 3 \mathrm{~A}$ & $1.283(13)$ & $\mathrm{C} 10 \mathrm{~A}-\mathrm{C} 11 \mathrm{~A}$ & $1.390(5)$ \\
\hline $\mathrm{N} 2-\mathrm{C} 9$ & $1.326(7)$ & $\mathrm{C} 11 \mathrm{~A}-\mathrm{C} 12 \mathrm{~A}$ & $1.385(4)$ \\
\hline $\mathrm{N} 2-\mathrm{C} 13$ & $1.320(5)$ & $\mathrm{C} 11 \mathrm{~A}-\mathrm{C} 14 \mathrm{~A}$ & $1.472(4)$ \\
\hline N3-C19 & $1.305(13)$ & $\mathrm{C} 12 \mathrm{~A}-\mathrm{H} 12 \mathrm{~A}$ & 0.9500 \\
\hline C9-H9 & 0.9500 & $\mathrm{C} 12 \mathrm{~A}-\mathrm{C} 13 \mathrm{~A}$ & $1.406(6)$ \\
\hline $\mathrm{C} 9-\mathrm{C} 10$ & $1.392(3)$ & $\mathrm{C} 13 \mathrm{~A}-\mathrm{H} 13 \mathrm{~A}$ & 0.9500 \\
\hline $\mathrm{C} 10-\mathrm{H} 10$ & 0.9500 & $\mathrm{C} 14 \mathrm{~A}-\mathrm{H} 14 \mathrm{~A}$ & 0.9500 \\
\hline $\mathrm{C} 10-\mathrm{C} 11$ & $1.386(3)$ & $\mathrm{C} 14 \mathrm{~A}-\mathrm{C} 15 \mathrm{~A}$ & $1.329(4)$ \\
\hline $\mathrm{C} 11-\mathrm{C} 12$ & $1.397(3)$ & $\mathrm{C} 15 \mathrm{~A}-\mathrm{H} 15 \mathrm{~A}$ & 0.9500 \\
\hline $\mathrm{C} 11-\mathrm{C} 14$ & $1.472(2)$ & $\mathrm{C} 15 \mathrm{~A}-\mathrm{C} 16 \mathrm{~A}$ & $1.469(5)$ \\
\hline $\mathrm{C} 7-\mathrm{O} 2-\mathrm{H} 2$ & $107.5(11)$ & $\mathrm{C} 15-\mathrm{C} 14-\mathrm{C} 11$ & $125.02(18)$ \\
\hline $\mathrm{C} 8-\mathrm{O} 4-\mathrm{H} 4$ & $111.7(11)$ & $\mathrm{C} 15-\mathrm{C} 14-\mathrm{H} 14$ & 117.5 \\
\hline $\mathrm{H} 1 \mathrm{~A}-\mathrm{N} 1-\mathrm{H} 1 \mathrm{~B}$ & $116.4(15)$ & $\mathrm{C} 14-\mathrm{C} 15-\mathrm{H} 15$ & 116.7 \\
\hline $\mathrm{C} 1-\mathrm{N} 1-\mathrm{H} 1 \mathrm{~A}$ & $119.4(11)$ & $\mathrm{C} 14-\mathrm{C} 15-\mathrm{C} 16$ & $126.5(2)$ \\
\hline $\mathrm{C} 1-\mathrm{N} 1-\mathrm{H} 1 \mathrm{~B}$ & $116.6(10)$ & $\mathrm{C} 16-\mathrm{C} 15-\mathrm{H} 15$ & 116.7 \\
\hline $\mathrm{N} 1-\mathrm{C} 1-\mathrm{C} 2$ & $121.17(9)$ & $\mathrm{C} 17-\mathrm{C} 16-\mathrm{C} 15$ & 123.3 \\
\hline $\mathrm{N} 1-\mathrm{C} 1-\mathrm{C} 6$ & $120.72(9)$ & $\mathrm{C} 20-\mathrm{C} 16-\mathrm{C} 15$ & $118.4(3)$ \\
\hline $\mathrm{C} 6-\mathrm{C} 1-\mathrm{C} 2$ & $118.07(9)$ & $\mathrm{C} 20-\mathrm{C} 16-\mathrm{C} 17$ & $118.3(4)$ \\
\hline $\mathrm{C} 1-\mathrm{C} 2-\mathrm{H} 2 \mathrm{~A}$ & 119.5 & $\mathrm{C} 18-\mathrm{C} 17-\mathrm{H} 17$ & 119.2 \\
\hline $\mathrm{C} 3-\mathrm{C} 2-\mathrm{C} 1$ & $121.01(9)$ & $\mathrm{C} 16-\mathrm{C} 17-\mathrm{C} 18$ & $121.5(4)$ \\
\hline $\mathrm{C} 3-\mathrm{C} 2-\mathrm{H} 2 \mathrm{~A}$ & 119.5 & $\mathrm{C} 16-\mathrm{C} 17-\mathrm{H} 17$ & 119.2 \\
\hline $\mathrm{C} 2-\mathrm{C} 3-\mathrm{C} 4$ & $120.80(9)$ & $\mathrm{N} 3-\mathrm{C} 19-\mathrm{H} 19$ & 118.6 \\
\hline $\mathrm{C} 2-\mathrm{C} 3-\mathrm{C} 8$ & $117.74(8)$ & $\mathrm{N} 3-\mathrm{C} 19-\mathrm{C} 20$ & $122.9(6)$ \\
\hline $\mathrm{C} 4-\mathrm{C} 3-\mathrm{C} 8$ & $121.44(9)$ & $\mathrm{C} 20-\mathrm{C} 19-\mathrm{H} 19$ & 118.6 \\
\hline $\mathrm{C} 3-\mathrm{C} 4-\mathrm{H} 4 \mathrm{~A}$ & 120.9 & $\mathrm{C} 16-\mathrm{C} 20-\mathrm{H} 20$ & 120.4 \\
\hline $\mathrm{C} 3-\mathrm{C} 4-\mathrm{C} 5$ & $118.24(9)$ & $\mathrm{C} 19-\mathrm{C} 20-\mathrm{C} 16$ & $119.2(4)$ \\
\hline $\mathrm{C} 5-\mathrm{C} 4-\mathrm{H} 4 \mathrm{~A}$ & 120.9 & $\mathrm{C} 19-\mathrm{C} 20-\mathrm{H} 20$ & 120.4 \\
\hline $\mathrm{C} 4-\mathrm{C} 5-\mathrm{C} 7$ & $122.17(9)$ & $\mathrm{C} 18-\mathrm{C} 17 \mathrm{~A}-\mathrm{H} 17 \mathrm{~A}$ & 122.4 \\
\hline $\mathrm{C} 6-\mathrm{C} 5-\mathrm{C} 4$ & $120.90(9)$ & $\mathrm{C} 18-\mathrm{C} 17 \mathrm{~A}-\mathrm{C} 16 \mathrm{~A}$ & $115.3(5)$ \\
\hline $\mathrm{C} 6-\mathrm{C} 5-\mathrm{C} 7$ & $116.93(8)$ & $\mathrm{C} 16 \mathrm{~A}-\mathrm{C} 17 \mathrm{~A}-\mathrm{H} 17 \mathrm{~A}$ & 122.4 \\
\hline $\mathrm{C} 1-\mathrm{C} 6-\mathrm{H} 6$ & 119.5 & $\mathrm{~N} 2 \mathrm{~A}-\mathrm{C} 9 \mathrm{~A}-\mathrm{H} 9 \mathrm{~A}$ & 117.7 \\
\hline $\mathrm{C} 5-\mathrm{C} 6-\mathrm{C} 1$ & $120.93(9)$ & $\mathrm{N} 2 \mathrm{~A}-\mathrm{C} 9 \mathrm{~A}-\mathrm{C} 10 \mathrm{~A}$ & $124.5(5)$ \\
\hline $\mathrm{C} 5-\mathrm{C} 6-\mathrm{H} 6$ & 119.5 & $\mathrm{C} 10 \mathrm{~A}-\mathrm{C} 9 \mathrm{~A}-\mathrm{H} 9 \mathrm{~A}$ & 117.7 \\
\hline $\mathrm{O} 1-\mathrm{C} 7-\mathrm{O} 2$ & $123.09(9)$ & $\mathrm{C} 19 \mathrm{~A}-\mathrm{C} 20 \mathrm{~A}-\mathrm{H} 20 \mathrm{~A}$ & 120.4 \\
\hline $\mathrm{O} 1-\mathrm{C} 7-\mathrm{C} 5$ & $121.82(9)$ & $\mathrm{C} 16 \mathrm{~A}-\mathrm{C} 20 \mathrm{~A}-\mathrm{H} 20 \mathrm{~A}$ & 120.4 \\
\hline $\mathrm{O} 2-\mathrm{C} 7-\mathrm{C} 5$ & $115.09(8)$ & $\mathrm{C} 16 \mathrm{~A}-\mathrm{C} 20 \mathrm{~A}-\mathrm{C} 19 \mathrm{~A}$ & $119.2(6)$ \\
\hline $\mathrm{O} 3-\mathrm{C} 8-\mathrm{O} 4$ & $123.31(9)$ & $\mathrm{C} 13 \mathrm{~A}-\mathrm{N} 2 \mathrm{~A}-\mathrm{C} 9 \mathrm{~A}$ & $114.3(9)$ \\
\hline $\mathrm{O} 3-\mathrm{C} 8-\mathrm{C} 3$ & $122.38(9)$ & $\mathrm{C} 18-\mathrm{N} 3 \mathrm{~A}-\mathrm{C} 19 \mathrm{~A}$ & $107.5(10)$ \\
\hline $\mathrm{O} 4-\mathrm{C} 8-\mathrm{C} 3$ & $114.30(8)$ & $\mathrm{C} 20 \mathrm{~A}-\mathrm{C} 19 \mathrm{~A}-\mathrm{H} 19 \mathrm{~A}$ & 117.5 \\
\hline $\mathrm{N} 3-\mathrm{C} 18-\mathrm{H} 18$ & 122.5 & $\mathrm{~N} 3 \mathrm{~A}-\mathrm{C} 19 \mathrm{~A}-\mathrm{C} 20 \mathrm{~A}$ & $125.0(9)$ \\
\hline $\mathrm{N} 3-\mathrm{C} 18-\mathrm{C} 17$ & $115.0(5)$ & $\mathrm{N} 3 \mathrm{~A}-\mathrm{C} 19 \mathrm{~A}-\mathrm{H} 19 \mathrm{~A}$ & 117.5 \\
\hline $\mathrm{C} 17-\mathrm{C} 18-\mathrm{H} 18$ & 122.5 & $\mathrm{C} 9 \mathrm{~A}-\mathrm{C} 10 \mathrm{~A}-\mathrm{H} 10 \mathrm{~A}$ & 120.1 \\
\hline $\mathrm{C} 17 \mathrm{~A}-\mathrm{C} 18-\mathrm{H} 18 \mathrm{~A}$ & 111.8 & $\mathrm{C} 9 \mathrm{~A}-\mathrm{C} 10 \mathrm{~A}-\mathrm{C} 11 \mathrm{~A}$ & $119.9(3)$ \\
\hline
\end{tabular}




\begin{tabular}{|c|c|c|c|}
\hline $\mathrm{N} 3 \mathrm{~A}-\mathrm{C} 18-\mathrm{H} 18 \mathrm{~A}$ & 111.8 & $\mathrm{C} 11 \mathrm{~A}-\mathrm{C} 10 \mathrm{~A}-\mathrm{H} 10 \mathrm{~A}$ & 120.1 \\
\hline $\mathrm{N} 3 \mathrm{~A}-\mathrm{C} 18-\mathrm{C} 17 \mathrm{~A}$ & $136.5(8)$ & $\mathrm{C} 10 \mathrm{~A}-\mathrm{C} 11 \mathrm{~A}-\mathrm{C} 14 \mathrm{~A}$ & $118.9(3)$ \\
\hline $\mathrm{C} 13-\mathrm{N} 2-\mathrm{C} 9$ & $119.0(5)$ & $\mathrm{C} 12 \mathrm{~A}-\mathrm{C} 11 \mathrm{~A}-\mathrm{C} 10 \mathrm{~A}$ & $117.2(2)$ \\
\hline $\mathrm{C} 19-\mathrm{N} 3-\mathrm{C} 18$ & $123.1(6)$ & $\mathrm{C} 12 \mathrm{~A}-\mathrm{C} 11 \mathrm{~A}-\mathrm{C} 14 \mathrm{~A}$ & $123.8(3)$ \\
\hline $\mathrm{N} 2-\mathrm{C} 9-\mathrm{H} 9$ & 118.9 & $\mathrm{C} 11 \mathrm{~A}-\mathrm{C} 12 \mathrm{~A}-\mathrm{H} 12 \mathrm{~A}$ & 120.7 \\
\hline $\mathrm{N} 2-\mathrm{C} 9-\mathrm{C} 10$ & $122.3(3)$ & $\mathrm{C} 11 \mathrm{~A}-\mathrm{C} 12 \mathrm{~A}-\mathrm{C} 13 \mathrm{~A}$ & $118.6(3)$ \\
\hline $\mathrm{C} 10-\mathrm{C} 9-\mathrm{H} 9$ & 118.9 & $\mathrm{C} 13 \mathrm{~A}-\mathrm{C} 12 \mathrm{~A}-\mathrm{H} 12 \mathrm{~A}$ & 120.7 \\
\hline $\mathrm{C} 9-\mathrm{C} 10-\mathrm{H} 10$ & 120.3 & $\mathrm{~N} 2 \mathrm{~A}-\mathrm{C} 13 \mathrm{~A}-\mathrm{C} 12 \mathrm{~A}$ & $125.3(6)$ \\
\hline $\mathrm{C} 11-\mathrm{C} 10-\mathrm{C} 9$ & $119.4(2)$ & $\mathrm{N} 2 \mathrm{~A}-\mathrm{C} 13 \mathrm{~A}-\mathrm{H} 13 \mathrm{~A}$ & 117.4 \\
\hline $\mathrm{C} 11-\mathrm{C} 10-\mathrm{H} 10$ & 120.3 & $\mathrm{C} 12 \mathrm{~A}-\mathrm{C} 13 \mathrm{~A}-\mathrm{H} 13 \mathrm{~A}$ & 117.4 \\
\hline $\mathrm{C} 10-\mathrm{C} 11-\mathrm{C} 12$ & $117.31(16)$ & $\mathrm{C} 11 \mathrm{~A}-\mathrm{C} 14 \mathrm{~A}-\mathrm{H} 14 \mathrm{~A}$ & 117.4 \\
\hline $\mathrm{C} 10-\mathrm{C} 11-\mathrm{C} 14$ & $123.2(2)$ & $\mathrm{C} 15 \mathrm{~A}-\mathrm{C} 14 \mathrm{~A}-\mathrm{C} 11 \mathrm{~A}$ & $125.2(3)$ \\
\hline $\mathrm{C} 12-\mathrm{C} 11-\mathrm{C} 14$ & $119.4(2)$ & $\mathrm{C} 15 \mathrm{~A}-\mathrm{C} 14 \mathrm{~A}-\mathrm{H} 14 \mathrm{~A}$ & 117.4 \\
\hline $\mathrm{C} 11-\mathrm{C} 12-\mathrm{H} 12$ & 120.4 & $\mathrm{C} 14 \mathrm{~A}-\mathrm{C} 15 \mathrm{~A}-\mathrm{H} 15 \mathrm{~A}$ & 117.3 \\
\hline $\mathrm{C} 13-\mathrm{C} 12-\mathrm{C} 11$ & $119.2(2)$ & $\mathrm{C} 14 \mathrm{~A}-\mathrm{C} 15 \mathrm{~A}-\mathrm{C} 16 \mathrm{~A}$ & $125.3(3)$ \\
\hline $\mathrm{C} 13-\mathrm{C} 12-\mathrm{H} 12$ & 120.4 & $\mathrm{C} 16 \mathrm{~A}-\mathrm{C} 15 \mathrm{~A}-\mathrm{H} 15 \mathrm{~A}$ & 117.3 \\
\hline $\mathrm{N} 2-\mathrm{C} 13-\mathrm{C} 12$ & $122.7(4)$ & $\mathrm{C} 17 \mathrm{~A}-\mathrm{C} 16 \mathrm{~A}-\mathrm{C} 15 \mathrm{~A}$ & $120.1(5)$ \\
\hline $\mathrm{N} 2-\mathrm{C} 13-\mathrm{H} 13$ & 118.6 & $\mathrm{C} 20 \mathrm{~A}-\mathrm{C} 16 \mathrm{~A}-\mathrm{C} 17 \mathrm{~A}$ & $116.4(5)$ \\
\hline $\mathrm{C} 12-\mathrm{C} 13-\mathrm{H} 13$ & 118.6 & $\mathrm{C} 20 \mathrm{~A}-\mathrm{C} 16 \mathrm{~A}-\mathrm{C} 15 \mathrm{~A}$ & $123.5(4)$ \\
\hline $\mathrm{C} 11-\mathrm{C} 14-\mathrm{H} 14$ & 117.5 & & \\
\hline $\mathrm{N} 1-\mathrm{C} 1-\mathrm{C} 2-\mathrm{C} 3$ & $178.19(10)$ & $\mathrm{C} 11-\mathrm{C} 14-\mathrm{C} 15-\mathrm{C} 16$ & $177.6(2)$ \\
\hline $\mathrm{N} 1-\mathrm{C} 1-\mathrm{C} 6-\mathrm{C} 5$ & $-179.82(10)$ & $\mathrm{C} 12-\mathrm{C} 11-\mathrm{C} 14-\mathrm{C} 15$ & $-173.6(2)$ \\
\hline $\mathrm{C} 1-\mathrm{C} 2-\mathrm{C} 3-\mathrm{C} 4$ & $1.71(16)$ & $\mathrm{C} 13-\mathrm{N} 2-\mathrm{C} 9-\mathrm{C} 10$ & $-0.7(9)$ \\
\hline $\mathrm{C} 1-\mathrm{C} 2-\mathrm{C} 3-\mathrm{C} 8$ & $-176.64(10)$ & $\mathrm{C} 14-\mathrm{C} 11-\mathrm{C} 12-\mathrm{C} 13$ & $174.4(3)$ \\
\hline $\mathrm{C} 2-\mathrm{C} 1-\mathrm{C} 6-\mathrm{C} 5$ & $-2.05(16)$ & $\mathrm{C} 14-\mathrm{C} 15-\mathrm{C} 16-\mathrm{C} 17$ & $-10.0(5)$ \\
\hline $\mathrm{C} 2-\mathrm{C} 3-\mathrm{C} 4-\mathrm{C} 5$ & $-2.20(16)$ & $\mathrm{C} 14-\mathrm{C} 15-\mathrm{C} 16-\mathrm{C} 20$ & $170.8(3)$ \\
\hline $\mathrm{C} 2-\mathrm{C} 3-\mathrm{C} 8-\mathrm{O} 3$ & $-7.84(17)$ & $\mathrm{C} 15-\mathrm{C} 16-\mathrm{C} 17-\mathrm{C} 18$ & $-177.6(3)$ \\
\hline $\mathrm{C} 2-\mathrm{C} 3-\mathrm{C} 8-\mathrm{O} 4$ & $171.27(10)$ & $\mathrm{C} 15-\mathrm{C} 16-\mathrm{C} 20-\mathrm{C} 19$ & $177.1(5)$ \\
\hline $\mathrm{C} 3-\mathrm{C} 4-\mathrm{C} 5-\mathrm{C} 6$ & $0.59(16)$ & $\mathrm{C} 17-\mathrm{C} 18-\mathrm{N} 3-\mathrm{C} 19$ & $-1.1(12)$ \\
\hline $\mathrm{C} 3-\mathrm{C} 4-\mathrm{C} 5-\mathrm{C} 7$ & $-179.15(10)$ & $\mathrm{C} 17-\mathrm{C} 18-\mathrm{C} 17 \mathrm{~A}-\mathrm{C} 16 \mathrm{~A}$ & $1.4(17)$ \\
\hline $\mathrm{C} 4-\mathrm{C} 3-\mathrm{C} 8-\mathrm{O} 3$ & $173.81(11)$ & $\mathrm{C} 17-\mathrm{C} 18-\mathrm{N} 3 \mathrm{~A}-\mathrm{C} 19 \mathrm{~A}$ & $-3.8(15)$ \\
\hline $\mathrm{C} 4-\mathrm{C} 3-\mathrm{C} 8-\mathrm{O} 4$ & $-7.07(15)$ & $\mathrm{C} 17-\mathrm{C} 16-\mathrm{C} 20-\mathrm{C} 19$ & $-2.0(7)$ \\
\hline $\mathrm{C} 4-\mathrm{C} 5-\mathrm{C} 6-\mathrm{C} 1$ & $1.56(16)$ & $\mathrm{C} 20-\mathrm{C} 16-\mathrm{C} 17-\mathrm{C} 18$ & $1.5(7)$ \\
\hline $\mathrm{C} 4-\mathrm{C} 5-\mathrm{C} 7-\mathrm{O} 1$ & $165.59(11)$ & $\mathrm{C} 17 \mathrm{~A}-\mathrm{C} 18-\mathrm{N} 3-\mathrm{C} 19$ & $-0.5(14)$ \\
\hline $\mathrm{C} 4-\mathrm{C} 5-\mathrm{C} 7-\mathrm{O} 2$ & $-14.70(15)$ & $\mathrm{C} 17 \mathrm{~A}-\mathrm{C} 18-\mathrm{C} 17-\mathrm{C} 16$ & $-179(3)$ \\
\hline $\mathrm{C} 6-\mathrm{C} 1-\mathrm{C} 2-\mathrm{C} 3$ & $0.43(16)$ & $\mathrm{C} 17 \mathrm{~A}-\mathrm{C} 18-\mathrm{N} 3 \mathrm{~A}-\mathrm{C} 19 \mathrm{~A}$ & $-4.3(18)$ \\
\hline $\mathrm{C} 6-\mathrm{C} 5-\mathrm{C} 7-\mathrm{O} 1$ & $-14.15(16)$ & $\mathrm{C} 9 \mathrm{~A}-\mathrm{N} 2 \mathrm{~A}-\mathrm{C} 13 \mathrm{~A}-\mathrm{C} 12 \mathrm{~A}$ & $3.1(13)$ \\
\hline $\mathrm{C} 6-\mathrm{C} 5-\mathrm{C} 7-\mathrm{O} 2$ & $165.55(10)$ & $\mathrm{C} 9 \mathrm{~A}-\mathrm{C} 10 \mathrm{~A}-\mathrm{C} 11 \mathrm{~A}-\mathrm{C} 12 \mathrm{~A}$ & $2.7(4)$ \\
\hline $\mathrm{C} 7-\mathrm{C} 5-\mathrm{C} 6-\mathrm{C} 1$ & $-178.69(10)$ & $\mathrm{C} 9 \mathrm{~A}-\mathrm{C} 10 \mathrm{~A}-\mathrm{C} 11 \mathrm{~A}-\mathrm{C} 14 \mathrm{~A}$ & $-174.9(3)$ \\
\hline $\mathrm{C} 8-\mathrm{C} 3-\mathrm{C} 4-\mathrm{C} 5$ & $176.09(10)$ & $\mathrm{N} 2 \mathrm{~A}-\mathrm{C} 9 \mathrm{~A}-\mathrm{C} 10 \mathrm{~A}-\mathrm{C} 11 \mathrm{~A}$ & $1.6(8)$ \\
\hline $\mathrm{C} 18-\mathrm{N} 3-\mathrm{C} 19-\mathrm{C} 20$ & $0.6(15)$ & $\mathrm{N} 3 \mathrm{~A}-\mathrm{C} 18-\mathrm{N} 3-\mathrm{C} 19$ & $-159(11)$ \\
\hline $\mathrm{C} 18-\mathrm{C} 17 \mathrm{~A}-\mathrm{C} 16 \mathrm{~A}-\mathrm{C} 20 \mathrm{~A}$ & $0.9(9)$ & $\mathrm{N} 3 \mathrm{~A}-\mathrm{C} 18-\mathrm{C} 17-\mathrm{C} 16$ & $2.5(10)$ \\
\hline $\mathrm{C} 18-\mathrm{C} 17 \mathrm{~A}-\mathrm{C} 16 \mathrm{~A}-\mathrm{C} 15 \mathrm{~A}$ & $-177.3(5)$ & $\mathrm{N} 3 \mathrm{~A}-\mathrm{C} 18-\mathrm{C} 17 \mathrm{~A}-\mathrm{C} 16 \mathrm{~A}$ & $2.8(15)$ \\
\hline $\mathrm{C} 18-\mathrm{N} 3 \mathrm{~A}-\mathrm{C} 19 \mathrm{~A}-\mathrm{C} 20 \mathrm{~A}$ & $2(2)$ & $\mathrm{C} 19 \mathrm{~A}-\mathrm{C} 20 \mathrm{~A}-\mathrm{C} 16 \mathrm{~A}-\mathrm{C} 17 \mathrm{~A}$ & $-2.1(11)$ \\
\hline $\mathrm{N} 2-\mathrm{C} 9-\mathrm{C} 10-\mathrm{C} 11$ & $-2.3(6)$ & $\mathrm{C} 19 \mathrm{~A}-\mathrm{C} 20 \mathrm{~A}-\mathrm{C} 16 \mathrm{~A}-\mathrm{C} 15 \mathrm{~A}$ & $176.0(8)$ \\
\hline $\mathrm{N} 3-\mathrm{C} 18-\mathrm{C} 17-\mathrm{C} 16$ & $0.0(8)$ & $\mathrm{C} 10 \mathrm{~A}-\mathrm{C} 9 \mathrm{~A}-\mathrm{N} 2 \mathrm{~A}-\mathrm{C} 13 \mathrm{~A}$ & $-4.4(12)$ \\
\hline $\mathrm{N} 3-\mathrm{C} 18-\mathrm{C} 17 \mathrm{~A}-\mathrm{C} 16 \mathrm{~A}$ & $-0.3(12)$ & $\mathrm{C} 10 \mathrm{~A}-\mathrm{C} 11 \mathrm{~A}-\mathrm{C} 12 \mathrm{~A}-\mathrm{C} 13 \mathrm{~A}$ & $-3.8(5)$ \\
\hline
\end{tabular}




$\begin{array}{llll}\mathrm{N} 3-\mathrm{C} 18-\mathrm{N} 3 \mathrm{~A}-\mathrm{C} 19 \mathrm{~A} & 19(9) & \mathrm{C} 10 \mathrm{~A}-\mathrm{C} 11 \mathrm{~A}-\mathrm{C} 14 \mathrm{~A}-\mathrm{C} 15 \mathrm{~A} & 168.9(3) \\ \mathrm{N} 3-\mathrm{C} 19-\mathrm{C} 20-\mathrm{C} 16 & 1.0(12) & \mathrm{C} 11 \mathrm{~A}-\mathrm{C} 12 \mathrm{~A}-\mathrm{C} 13 \mathrm{~A}-\mathrm{N} 2 \mathrm{~A} & 0.9(9) \\ \mathrm{C} 9-\mathrm{N} 2-\mathrm{C} 13-\mathrm{C} 12 & 1.9(9) & \mathrm{C} 11 \mathrm{~A}-\mathrm{C} 14 \mathrm{~A}-\mathrm{C} 15 \mathrm{~A}-\mathrm{C} 16 \mathrm{~A} & -177.1(3) \\ \mathrm{C} 9-\mathrm{C} 10-\mathrm{C} 11-\mathrm{C} 12 & 4.0(3) & \mathrm{C} 12 \mathrm{~A}-\mathrm{C} 11 \mathrm{~A}-\mathrm{C} 14 \mathrm{~A}-\mathrm{C} 15 \mathrm{~A} & -8.5(5) \\ \mathrm{C} 9-\mathrm{C} 10-\mathrm{C} 11-\mathrm{C} 14 & -173.2(2) & \mathrm{C} 14 \mathrm{~A}-\mathrm{C} 11 \mathrm{~A}-\mathrm{C} 12 \mathrm{~A}-\mathrm{C} 13 \mathrm{~A} & 173.6(4) \\ \mathrm{C} 10-\mathrm{C} 11-\mathrm{C} 12-\mathrm{C} 13 & -2.9(3) & \mathrm{C} 14 \mathrm{~A}-\mathrm{C} 15 \mathrm{~A}-\mathrm{C} 16 \mathrm{~A}-\mathrm{C} 17 \mathrm{~A} & -172.8(5) \\ \mathrm{C} 10-\mathrm{C} 11-\mathrm{C} 14-\mathrm{C} 15 & 3.5(3) & \mathrm{C} 14 \mathrm{~A}-\mathrm{C} 15 \mathrm{~A}-\mathrm{C} 16 \mathrm{~A}-\mathrm{C} 20 \mathrm{~A} & 9.1(6) \\ \mathrm{C} 11-\mathrm{C} 12-\mathrm{C} 13-\mathrm{N} 2 & -0.1(6) & \mathrm{C} 16 \mathrm{~A}-\mathrm{C} 20 \mathrm{~A}-\mathrm{C} 19 \mathrm{~A}-\mathrm{N} 3 \mathrm{~A} & 0.5(19)\end{array}$

Hydrogen-bond geometry $\left(\AA,{ }^{\circ}\right)$

\begin{tabular}{lllll}
\hline$D-\mathrm{H} \cdots A$ & $D-\mathrm{H}$ & $\mathrm{H} \cdots A$ & $D \cdots A$ & $D-\mathrm{H} \cdots A$ \\
\hline $\mathrm{N} 1-\mathrm{H} 1 A \cdots \mathrm{O} 1^{\mathrm{i}}$ & $0.899(17)$ & $2.062(17)$ & $2.9540(13)$ & $171.0(15)$ \\
$\mathrm{N} 1-\mathrm{H} 1 B \cdots \mathrm{O} 3^{\mathrm{ii}}$ & $0.894(17)$ & $2.157(17)$ & $3.0500(13)$ & $178.6(13)$ \\
$\mathrm{O} 2-\mathrm{H} 2 \cdots \mathrm{N} 3^{\mathrm{iii}}$ & $0.989(19)$ & $1.70(2)$ & $2.688(8)$ & $173.4(18)$ \\
$\mathrm{O} 2-\mathrm{H} 2 \cdots \mathrm{N} 3 A^{\mathrm{iii}}$ & $0.989(19)$ & $1.63(2)$ & $2.619(12)$ & $177(2)$ \\
$\mathrm{O} 4-\mathrm{H} 4 \cdots \mathrm{N} 2^{\text {iv }}$ & $0.98(2)$ & $1.72(2)$ & $2.702(7)$ & $173.2(19)$ \\
$\mathrm{O} 4-\mathrm{H} 4 \cdots \mathrm{N} 2 A^{\text {iv }}$ & $0.98(2)$ & $1.59(2)$ & $2.566(11)$ & $175(2)$
\end{tabular}

Symmetry codes: (i) $-x+1 / 2, y-1 / 2,-z+1 / 2$; (ii) $-x+1 / 2, y+1 / 2,-z+1 / 2$; (iii) $-x+1,-y+2,-z+1$; (iv) $x-1 / 2,-y+1 / 2, z+1 / 2$. 